\title{
Reconciliação Terapêutica na Admissão de um Serviço de Medicina Interna: Estudo-Piloto
}

\author{
Medication Reconciliation During Admission to an Internal \\ Medicine Department: A Pilot Study
}

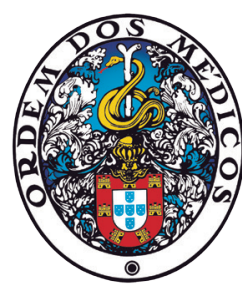

Thaís COSTA E SILVA ${ }^{1}$, Patrícia DIAS ${ }^{2}$, Catarina ALVES E CUNHA ${ }^{3}$, José FEIO ${ }^{4}$, Marta LAVRADOR ${ }^{1,5}$, Joelizy OLIVEIRA ${ }^{1,6,7}$, Isabel Vitória FIGUEIREDO ${ }^{1,5}$, Marília João ROCHA ${ }^{4}$, Margarida CASTEL-BRANCO ${ }^{1,5}$ Acta Med Port 2022 Nov;35(11):798-806 - https://doi.org/10.20344/amp.16892

RESUMO

Introdução: A reconciliação terapêutica visa promover a segurança do doente por meio da redução de erros de medicação e eventos adversos decorrentes de discrepâncias de medicação na transição de cuidados. Foi nosso objetivo realizar um estudo-piloto de reconciliação terapêutica no momento da admissão hospitalar para, a partir dele, identificarmos os recursos necessários para a sua implementação na prática clínica.

Material e Métodos: Estudo-piloto com 100 doentes admitidos num serviço de Medicina Interna entre outubro e dezembro de 2019, com mais de 18 anos e a tomar cronicamente pelo menos um medicamento. A melhor história farmacoterapêutica possível foi obtida sistematicamente, com posterior identificação, classificação e resolução das discrepâncias.

Resultados: A amostra em estudo, em geral polimedicada e com múltiplas morbilidades, apresentou uma média de idades de 77,04 $\pm 13,74$ anos, sendo $67,0 \%$ do sexo masculino. Foram identificadas 791 discrepâncias e as intencionais (95,7\%) estavam documentadas em 50,9\% das situações. As dificuldades encontradas relacionaram-se principalmente com o acesso e a qualidade da informação terapêutica e com a dificuldade de comunicação entre os diversos profissionais de saúde. Os principais recursos prioritários identificados relacionaram-se com as categorias de processo, ferramentas e pessoal.

Conclusão: Os dados revelaram fragilidades nos registos clínicos disponíveis na interface dos cuidados primários/hospitalares. A otimização das fontes de dados, normalização e informatização do processo, atuação multidisciplinar e definição de grupos prioritários foram identificadas como oportunidades de otimização.

Palavras-chave: Cuidado Transicional; Erros de Medicação; Medicina Interna; Reconciliação de Medicamentos; Segurança do Doente

\section{ABSTRACT}

Introduction: The purpose of medication reconciliation is to promote patient safety by reducing medication errors and adverse events due to medication discrepancies in transition of care. The aim of this pilot study of medication reconciliation at the time of hospital admission was to identify the necessary resources for its implementation in clinical practice.

Material and Methods: Pilot study with 100 patients admitted to an Internal Medicine department between October and December 2019, aged 18 and over, and chronically taking at least one medicine. The best possible medication history was obtained systematically, with subsequent identification, classification and resolution of the discrepancies.

Results: The study sample, in general characterized by polypharmacy and by having multiple long-term conditions, presented a mean age of $77.04 \pm 13.74$ years, being $67.0 \%$ male. Overall, 791 discrepancies were identified. Intentional discrepancies were $95.7 \%$ and $50.9 \%$ of them were documented. The difficulties encountered were mainly related with the access and quality of therapeutic information and communication problems between different healthcare professionals. The key priority resources that were identified were related with the process, tools, and personnel categories.

Conclusion: The data revealed weaknesses in the clinical records available at the primary/hospital care interface. Optimization of data sources, standardization and informatization of the process, multidisciplinary approach and definition of priority groups were identified as opportunities for optimization.

Keywords: Internal Medicine; Medication Errors; Medication Reconciliation; Patient Safety; Transitional Care

\section{INTRODUÇÃO}

Segundo a Direção-Geral da Saúde (DGS), a reconciliação terapêutica define-se como sendo "um processo de análise da medicação de um doente, sempre que ocorrem alterações na medicação, com o objetivo de evitar discrepâncias, nomeadamente omissões, duplicações ou doses inadequadas, promovendo a adesão à medicação e con- tribuindo para a prevenção de incidentes relacionados com a medicação". ${ }^{1}$ Esta intervenção ganhou especial relevância com o aumento da esperança de vida global, que se reflete num aumento do número de utentes dos serviços de saúde com idade mais avançada, patologias múltiplas e polimedicação crónica.

1. Laboratório de Farmacologia e Cuidados Farmacêuticos. Faculdade de Farmácia. Universidade de Coimbra. Coimbra. Portugal.

2. Serviço de Medicina Interna. Centro Hospitalar e Universitário de Coimbra. Coimbra. Portugal.

3. Unidade de Farmacologia Clínica. Centro Hospitalar e Universitário de Coimbra. Coimbra. Portugal.

4. Serviços Farmacêuticos. Centro Hospitalar e Universitário de Coimbra. Coimbra. Portugal.

5. Instituto de Investigação Clínica e Biomédica de Coimbra. Coimbra. Portugal.

6. Fundação Capes. Ministério da Educação. Brasília. Brasil.

7. Centro de Documentação e Informação em Educação Superior. Ministério da Educação Superior e Pesquisa do Governo do Grão-Ducado de Luxemburgo. Luxemburgo.

$\triangle$ Autor correspondente: Margarida Castel-Branco. mmcb@ci.uc.pt

Recebido/Received: 20/07/2021 - Aceite/Accepted: 15/12/2021 - Publicado Online/Published Online: 04/03/2022 - Publicado/Published: 02/11/2022

Copyright $\odot$ Ordem dos Médicos 2022 
A reconciliação terapêutica consiste, portanto, na avaliação sistematizada de todos os medicamentos que estão a ser adicionados, alterados ou descontinuados a um doente na transição de cuidados, momento este que se configura como sendo o mais vulnerável para a ocorrência de erros e, consequentemente, o mais elegível para a implementação de estratégias que procurem preveni-los. ${ }^{2,3}$ Através da Norma 018/2016, a DGS estabeleceu que as instituições portuguesas prestadoras de cuidados de saúde devem promover a implementação da reconciliação terapêutica, de forma a normalizar a forma de comunicação entre os profissionais de saúde e garantir a informação essencial sobre o doente e a sua medicação. ${ }^{1}$

A reconciliação terapêutica tem como fundamento o princípio de que, partindo de uma lista atualizada e fidedigna do regime terapêutico atual, a informação farmacoterapêutica é transmitida de forma efetiva na continuidade dos cuidados, sendo sobre esta que o plano terapêutico vai ser construído e otimizado, à medida que a situação clínica o exija. Esta lista inicial, intitulada como best possible medication history (BPMH), possibilita à equipa clínica não só a visão terapêutica global do doente, como a construção facilitada do plano de alta hospitalar, reduzindo os riscos de que informações importantes possam não ser transmitidas de forma efetiva para o próximo serviço ou para o próprio doente. ${ }^{4}$

As discrepâncias podem ocorrer no momento da admissão, durante o internamento ou no momento da alta hospitalar. Numa revisão sobre a reconciliação terapêutica na transição do doente do hospital para os cuidados primários de saúde, a análise de 15 artigos abrangendo 6000 altas hospitalares identificou uma variação de $20 \%$ a $87 \%$ no número de doentes com discrepâncias relacionadas com a terapêutica, ${ }^{5}$ o que salienta a necessidade de tornar esta informação transversal a todo o sistema de saúde.

Apesar do seu contributo no fortalecimento das práticas de segurança do doente, estas intervenções desenvolvidas e implementadas na transição de cuidados exigem comunicação efetiva das informações, ao mesmo tempo que envolvem diferentes pessoas, profissionais, tecnologias, processos e serviços. ${ }^{6}$ Desta forma, os hospitais ainda encontram muitos desafios para aplicarem esta ferramenta na prática clínica, uma vez que a necessidade de recursos e a integração num fluxo de trabalho pré-existente obriga à conciliação de diferentes fatores clínicos, comportamentais e organizacionais.

Neste contexto, a falta do estabelecimento de medidas claras e da uniformização de conceitos entre os países da União Europeia faz com que ainda hoje iniciativas neste sentido sejam promovidas muitas vezes de forma individual e com adaptação de modelos internacionais. Assim, tendo em vista aprofundar este conhecimento a nível nacional, Oliveira e colaboradores (2020) avaliaram as potenciais contribuições das principais fontes de informação disponíveis em Portugal na obtenção da $\mathrm{BPMH}$, demonstrando a importância dos registos eletrónicos em saúde, representados nomeadamente pela plataforma de dados da saúde
(PDS), na maior exatidão da informação terapêutica, particularmente ao se considerar um período retrospetivo de seis meses ao internamento. ${ }^{7}$

$\mathrm{Na}$ continuidade deste trabalho, foi nosso objetivo realizar um estudo-piloto de Reconciliação Terapêutica no momento da admissão hospitalar para, a partir dele, identificarmos os recursos necessários para a sua implementação na prática clínica.

\section{MATERIAL E MÉTODOS}

Estudo-piloto, de natureza prospetiva, conduzido entre outubro e dezembro de 2019 no Serviço de Medicina Interna do Centro Hospitalar e Universitário de Coimbra (CHUC). O estudo foi aprovado pela comissão de ética da mesma instituição (CHUC-133-19) e todos os participantes assinaram um termo de consentimento informado.

Foram incluídos no estudo os primeiros 100 doentes admitidos no serviço de Medicina Interna com idade superior a 18 anos e a tomar, pelo menos, um medicamento no domicílio. Foram definidos como critérios de exclusão situações de impossibilidade de realização da entrevista, como sejam doentes sem condições de comunicação e ausência de representação por um familiar ou cuidador (como acontece nos casos sociais).

O ponto de transição escolhido foi a admissão, conforme previsto pela DGS, por ser o primeiro ponto crítico a ser considerado na implementação da reconciliação terapêutica ao nível dos Cuidados Hospitalares. ${ }^{1}$

O estudo realizou-se em várias etapas:

1. Recolha dos dados sociodemográficos e informações gerais. Preenchemos um formulário próprio com dados sociodemográficos do doente (sexo, idade, tipo de residência e índice de atividade básica de vida diária), hábitos (tabágicos, alcoólicos e autonomia na gestão dos medicamentos) e condições clínicas (comorbilidades, alergias e parâmetros clínicos). A recolha da informação foi realizada recorrendo ao processo informatizado do doente, por meio das aplicações informáticas SGCIM (sistema de gestão integrado do circuito do medicamento - módulo de prescrição), ALERT (software do serviço de urgência - dados de admissão, evolução clínica e prescrição) e SClínico Hospitalar (histórico dos episódios clínicos e evolução clínica atual). Também consultámos os processos clínicos físicos.

\section{Obtenção do perfil farmacoterapêutico pré-hospitalar e construção da BPMH. Além dos registos hospitalares,} foram consultados o Registo de Saúde Eletrónico (RSE) na Plataforma de Dados da Saúde (PDS) num período retrospetivo de seis meses a contar da data de admissão hospitalar, ${ }^{7}$ prescrições médicas de ambulatório, listas e sacos de medicação trazidos pelo doente ou das instituições de longa permanência, assim como demais prescrições, planos de alta e evoluções de internamentos anteriores. $\mathrm{Na}$ consulta à PDS foram verificados a disponibilização e o status de atualização das prescrições dos últimos seis meses e da lista de medicação crónica, assim como o histórico de dispensa dos medicamentos. 
Para finalizar esta etapa, o farmacêutico-investigador realizou uma entrevista semiestruturada a todos os participantes (doente/cuidador) com vista à confirmação dos dados obtidos, considerando-se esta informação como sendo a mais exata. Feita a confrontação dos dados obtidos na entrevista com, pelo menos, mais uma das fontes de dados citadas, construímos a BPMH, permitindo a identificação dos medicamentos de uso crónico pré-hospitalar (nome do medicamento, dose, forma farmacêutica, frequência e via de administração). Considerámos todos os medicamentos pré-hospitalares, com ou sem prescrição médica, bem como os fitoterapêuticos e chás medicinais. Também considerámos os medicamentos prescritos por tempo determinado cujo tratamento estivesse em curso na data de admissão. A polimedicação foi definida como sendo a toma de cinco ou mais fármacos.

3. Consulta da prescrição de internamento e identificação das discrepâncias. Uma vez obtida a BPMH, o investigador consultou a prescrição do internamento válida nas primeiras 24 horas de internamento de cada doente, recolhendo os dados referentes aos medicamentos. Por comparação da medicação instituída aquando da admissão com a informação relativa à medicação pré-hospitalar constante na BPMH identificaram-se as discrepâncias.

4. Classificação das discrepâncias. Uma vez identificadas, as discrepâncias foram classificadas de acordo com a sua intencionalidade e documentação. Além disso, foram ainda divididas por categorias e gravidade e identificadas as classes farmacoterapêuticas envolvidas. Compõem estas categorias a omissão ou adição de um medicamento, a substituição terapêutica, as alterações de dose, frequência, forma farmacêutica ou via de administração. ${ }^{8}$ Para os erros de medicação também foi considerada a categoria 'conciliação', que se refere às situações em que, mediante os parâmetros clínicos do doente na admissão, o medicamento habitual não deveria ter sido mantido ou ainda os casos em que o medicamento foi introduzido, mas já se encontrava suspenso anteriormente à admissão.

Discrepâncias intencionais (DI) - As DI podem ser subdivididas em 'documentadas' (quando a justificação da alteração está devidamente registada no processo clínico) ou 'não documentadas' (quando a justificação não está claramente documentada). ${ }^{4}$ Neste caso foram divididas em três categorias referentes à justificação da alteração: justificada por alteração nos parâmetros clínicos, por seguimento de diretrizes terapêuticas ou protocolos hospitalares ou por necessidade adicional de confirmação da justificação pelo médico prescritor.

Discrepâncias não intencionais (DNI) ou erros de medicação - A avaliação das DNI, além da categorização, foi realizada pelo critério de gravidade/potencial em causar danos. Foram avaliadas por um médico especialista em farmacologia clínica de maneira independente da unidade de estudo com o conhecimento apenas das informações necessárias para exercer o seu julgamento clínico. A ca- tegorização seguiu o modelo de gravidade de Buckley et al, que as considera 'sem significado clínico', 'clinicamente significativas', 'clinicamente graves' ou com 'risco de vida'. ${ }^{9}$

5. Comunicação das discrepâncias. No fim de todo o processo, as discrepâncias identificadas que requeriam avaliação foram comunicadas ao médico prescritor, por via presencial ou informática, para consequente resolução quando necessário.

Realizámos a análise descritiva quantitativa das fontes de dados utilizadas para obtenção da BPMH de cada doente, assim como a caracterização da idade, número de medicamentos e de comorbilidades, local de proveniência, forma de admissão, número de discrepâncias e sua classificação. As informações recolhidas foram introduzidas na base de dados do Microsoft Office Excel ${ }^{\circledR} 2019$ e realizada a análise através do software SPSS ${ }^{\circledR}$ 2019. Foram calculadas as frequências absolutas e relativas (percentagens) das variáveis analisadas, assim como as médias e desvios-padrão.

A reconciliação terapêutica efetuada foi também analisada do ponto de vista qualitativo tendo em vista a identificação dos recursos necessários para a sua implementação na admissão hospitalar, bem como a identificação de oportunidades de otimização dos recursos já existentes.

\section{RESULTADOS}

\section{Caracterização da população}

A população em estudo apresentou uma média de idade de $77,04 \pm 13,74$ anos (média \pm desvio-padrão), $80 \%$ com idade $\geq 65$ anos e $33 \%$ do sexo feminino (Tabela 1). O número de medicamentos pré-hospitalares foi de $7,72 \pm$ 3,01 medicamentos/doente, com variação de um a 14 medicamentos. A polimedicação foi identificada em $85 \%$ dos

Tabela 1 - Caracterização dos dados sociodemográficos da população em estudo $(n=100)$

\begin{tabular}{lc}
\hline Variáveis em estudo & Resultados \\
\hline Tamanho da amostra, $\mathrm{n}(\%)$ & $100(100 \%)$ \\
Idade, (média \pm DP) & $77,04 \pm 3,74$ \\
Sexo, $\mathrm{n}$ (\% feminino) & $33(33 \%)$ \\
Residência, $\mathrm{n}(\%)$ & \\
Domicílio & $67(67 \%)$ \\
Lar & $28(28 \%)$ \\
Unidade de Cuidados Continuados (UCC) & $5(5 \%)$ \\
Tipo de Admissão, $\mathrm{n}(\%)$ & \\
Serviço de Urgência & $94(94 \%)$ \\
Consultas ambulatoriais & $6(6 \%)$ \\
Índice Katz, n (\%) & \\
0 & $34(34 \%)$ \\
$1-2$ & $16(16 \%)$ \\
$3-4$ & $9(9 \%)$ \\
5 & $13(13 \%)$ \\
6 & $28(28 \%)$ \\
\hline
\end{tabular}


participantes, assim como um perfil comum de múltiplas comorbilidades (Tabela 2), tendo sido mais prevalentes a insuficiência respiratória, a hipertensão arterial e a diabetes mellitus tipo 2. O diagnóstico mais prevalente na admissão correspondeu às doenças do sistema respiratório (32\%), principalmente a pneumonia bacteriana (CID J15), seguida de doenças correspondentes ao sistema circulatório (16\%), geniturinário (14\%) e digestivo (12\%).

\section{Fontes de dados para obtenção da BPMH}

A construção da BPMH durou em média 41,80 \pm 8,40 minutos por doente. O tempo médio das entrevistas foi de $11,0 \pm 3,20$ minutos, sendo o restante tempo atribuído à consulta das demais fontes de dados. A entrevista teve de ser feita com acompanhamento de um familiar em $48 \%$ das situações; em $22 \%$ foi possível somente com o doente, e nos casos de doentes institucionalizados, em que o familiar não sabia fornecer a informação, esta foi confirmada com o lar. Foi consultada a lista de medicação fornecida pelo doente ou pelo lar em $49 \%$ dos participantes e o saco de medicação em $25 \%$.

Em $17 \%$ dos doentes não foi possível o acesso à PDS a nenhum dos três itens (prescrição dos últimos seis meses, lista de medicação crónica e histórico da dispensa dos medicamentos). Nos $83 \%$ dos doentes em que o acesso à PDS foi possível, o 'histórico de dispensa' foi o item que esteve sempre disponibilizado. No entanto, 54\% não continham o item 'registo da prescrição dos últimos seis meses' disponível e 70\%, apesar de terem a 'lista de medicação crónica' disponível, esta estava desatualizada em 59\% das situações quando comparada com a informação obtida na entrevista.

\section{Identificação e classificação das discrepâncias}

No total, foram identificadas 791 discrepâncias, tendo as intencionais $(95,7 \%)$ sido classificadas como documentadas em 50,9\% das situações. As discrepâncias intencionais não documentadas foram analisadas e divididas conforme justificadas por condições e parâmetros clínicos $(15,05 \%)$, por protocolos e diretrizes hospitalares $(43,55 \%)$ e por necessidade de confirmação adicional junto do médico $(41,40 \%)$. A categorização das discrepâncias (DI e DNI) está descrita nas Fig.s 1 e 2, respetivamente.

Foram identificadas $34 \mathrm{DNI}$ ou erros de medicação em 22 doentes. Na avaliação do potencial em causar danos, $55,88 \%$ foram avaliadas como não tendo significado clínico para o doente e envolveram principalmente a omissão de medicamentos analgésicos ou antidislipidémicos. As discrepâncias que foram classificadas com potencial de danos clinicamente significativos ou clinicamente graves contemplaram oito e dois doentes, respetivamente, e corresponderam por sua vez a $32,35 \%$ e $11,76 \%$ do total de DNI. As discrepâncias avaliadas como tendo potencial de danos clinicamente significativos foram classificadas nas categorias conciliação e na sua maioria omissão $(90,90 \%)$, esta segunda envolvendo fármacos ansiolíticos $(27,27 \%)$, antidepressivos $(18,18 \%)$, antianémicos $(18,18 \%)$, antigotosos $(18,18 \%)$, vasoprotetores $(9,09 \%)$ e inaladores adrenérgicos associados a corticoide $(9,09 \%)$. As discrepâncias avaliadas como clinicamente graves envolveram, por sua vez e na sua totalidade, fármacos de ação no aparelho cardiovascular, nomeadamente agentes betabloqueantes (50\%) e diuréticos (50\%). As categorias envolvidas foram omissão (50\%), alteração de dose (25\%) e conciliação (25\%).

Tabela 2 - Caracterização dos dados clínicos da população em estudo ( $n=100)$

\begin{tabular}{lc}
\hline Variáveis em estudo & Resultado \\
\hline Tamanho da amostra, $n(\%)$ & $100(100 \%)$ \\
Autonomia na gestão dos medicamentos, $n(\%)$ & $26(26 \%)$ \\
Doente & $38(38 \%)$ \\
Familiar/cuidador & $36(36 \%)$ \\
Profissional do lar/UCC/Centro de Dia & $7,72 \pm 3,01$ \\
Número de medicamentos pré-hospitalares, (média \pm DP) & $15(15 \%)$ \\
Número de medicamentos pré-hospitalares, $n(\%)$ & $56(56 \%)$ \\
$1-4$ & $29(29 \%)$ \\
$5-9$ & \\
$\geq 10$ & $8(8 \%)$ \\
Alergia a medicamentos, $n(\%)$ & $92(92 \%)$ \\
Sim & $7,80 \pm 2,53$ \\
Não & $10(10 \%)$ \\
Número de comorbilidades, (média \pm DP) & $67(67 \%)$ \\
Número de comorbilidades, $n(\%)$ & $23(23 \%)$ \\
$0-4$ & \\
$10-15$ & \\
\hline
\end{tabular}


Omissão

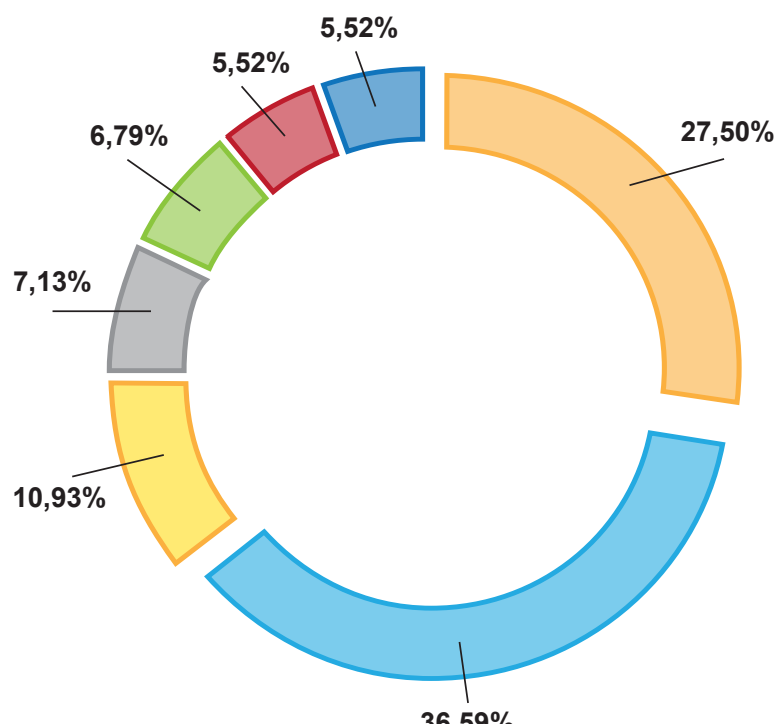

Figura 1 - Categorização das Discrepâncias Intencionais

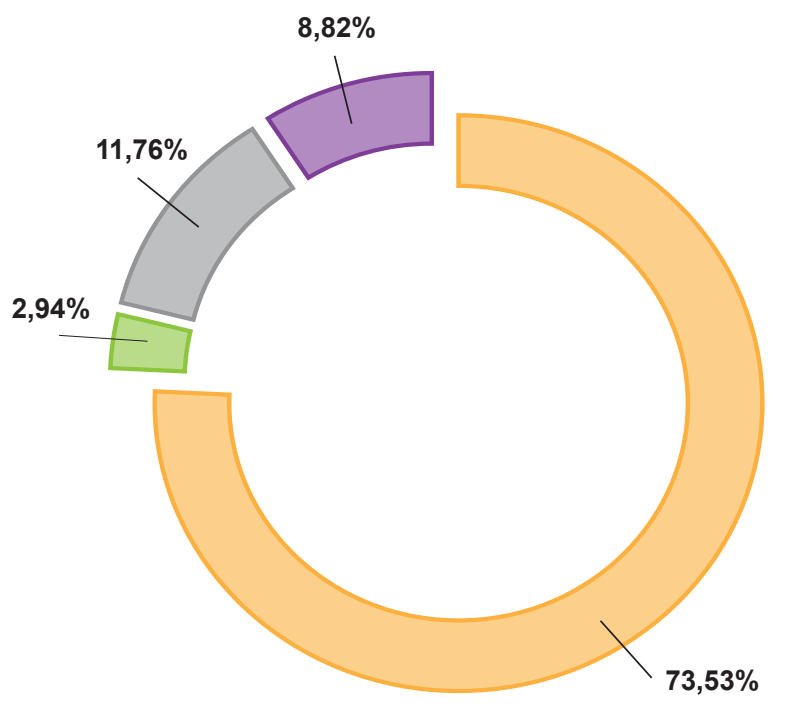

Figura 2 - Categorização das Discrepâncias Não Intencionais

Recursos necessários e oportunidades de otimização

As dificuldades identificadas durante o processo da reconciliação terapêutica englobaram falta de ferramentas informáticas que apoiem o processo, falta de padronização nos registos da informação terapêutica e suas alterações, dificuldades no acesso ao familiar ou instituição de saúde (tempo despendido para obtenção da entrevista, listas ou sacos de medicamentos) e falta de disponibilidade do médico responsável pelo doente (comunicação, feedback e resolução das discrepâncias).

Os recursos necessários para que a implementação da reconciliação terapêutica seja realizada de forma efetiva e sustentável na prática clínica foram divididos em seis cate-
Omissão

Frequência

Dose

Conciliação
Adição

Substituição terapêutica

Dose

Frequência

Via de administração

Forma farmacêutica gorias principais: regulamentação, processo, pessoal, gestão, ferramentas e formação. Falhas em um ou mais destes recursos implicam desafios de aplicabilidade da reconciliação terapêutica, o que automaticamente os configura como pontos de intervenção (Fig. 3). No presente estudo, com base nas dificuldades identificadas, os principais recursos envolveram principalmente as categorias de processo, ferramentas e pessoal.

\section{DISCUSSÃO}

Embora não seja responsável, sozinha, pela redução dos erros de medicação e pelo aumento da segurança do doente, a reconciliação terapêutica apresenta-se como a 


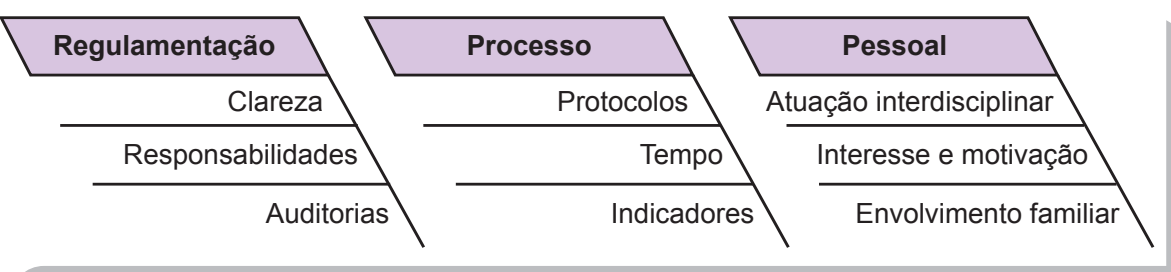

FALHAS

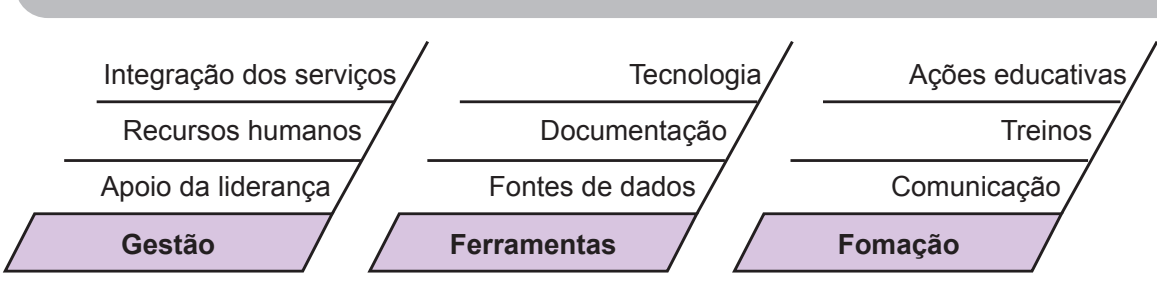

Desafios de aplicabilidade da Reconcilliação Terapêutica

Figura 3 - Recursos envolvidos no processo de Reconciliação Terapêutica

primeira e talvez a mais crítica peça do quebra-cabeças que envolve a gestão da informação terapêutica na transição de cuidados. ${ }^{10}$ Desta forma, para se poderem implementar planos de ações mais abrangentes, é preciso iniciar da base e construir passo-a-passo o caminho. Para isso, realizámos o estudo-piloto aqui apresentado, o que nos permitiu mapear os principais recursos necessários, com consequente identificação de oportunidades de otimização, definidas como estratégias e ações que diminuam as barreiras do processo de reconciliação e o tornem mais aplicável.

A caracterização da população estudada corrobora uma análise anterior realizada sobre a evolução do Serviço de Medicina Interna nos últimos 20 anos nesta mesma instituição: elevada prevalência de doentes idosos, com múltiplas patologias, polimedicação e pouca autonomia na gestão dos medicamentos, fatores estes que são comuns às unidades de medicina interna e que se configuram, segundo a literatura, como fatores de risco na transição de cuidados. ${ }^{12,13}$ Assim sendo, estes serviços tornam-se alvos potenciais para implementação da reconciliação terapêutica, que se apresenta como uma importante ferramenta de apoio com impacto na identificação de erros de medicação e consequente prevenção de eventos adversos relacionados com falhas de comunicação entre os serviços e entre o próprio doente e o serviço.

Quanto à análise das discrepâncias, 49,14\% das avaliadas como intencionais foram classificadas como não documentadas, pois não continham justificação da alteração no processo clínico do doente. Estas discrepâncias são consideradas falhas de documentação. Destas, 41,40\% não atendiam aos critérios de alteração intencional baseada nos parâmetros clínicos ou intercâmbio terapêutico, e necessitaram de esclarecimento adicional do prescritor, envolvendo algumas classes terapêuticas principais como antidislipidémicos e antianémicos. Apesar de não serem consideradas erros de medicação e muitas vezes não representarem um risco imediato à segurança do doente, discrepâncias não documentadas, mesmo que intencionais, influenciam os recursos necessários e resultados da re- conciliação terapêutica, pois podem gerar confusão, exigir esclarecimentos adicionais e levar a futuros erros de medicação, por exemplo, na alta hospitalar.4,14,15

De entre as categorias de discrepâncias intencionais identificadas, a adição de um novo medicamento (ex. introdução de agentes anti-infecciosos), a omissão de um medicamento prévio (ex. suspensão de anti-hipertensores orais) e a substituição terapêutica de um medicamento por outro para o mesmo objetivo terapêutico (ex. substituição de antidiabéticos orais por insulina subcutânea) foram as categorias mais frequentes. É importante a compreensão, quando se aplica a reconciliação terapêutica, de que alterações intencionais no regime terapêutico do doente muitas vezes se justificam pela condição clínica que o levou ao internamento, pelo plano terapêutico de resposta estabelecido e também pelo arsenal terapêutico disponível no hospital.

Quando há intencionalidade nas discrepâncias, o importante é que estas sejam registadas e possam ser consultadas e comunicadas de forma clara entre os profissionais e ao doente, de modo que, ao retornar ao seu quotidiano, este tenha a perceção de quais medicamentos foram descontinuados, alterados ou adicionados. É nisto que se encontra a importância da reconciliação, tanto na admissão como na alta, pois permite que a informação terapêutica seja longitudinal ao internamento e dê suporte à construção do plano de alta, com fortalecimento da adesão ao tratamento de forma correta pelo doente e melhor alcance dos objetivos clínicos estabelecidos.

Nas discrepâncias não intencionais, por sua vez, foi possível identificar a omissão de medicamentos (ex. medicamentos atuantes no sistema nervoso central e aparelho cardiovascular) como sendo a categoria mais frequente, o que é similar aos resultados encontrados na literatura. ${ }^{9,16,17}$ Isto ocorre quando há falha na reconciliação de um medicamento que o doente estava a tomar previamente e passa a não o tomar durante o internamento, o que pode estar relacionado com diversas razões que levam à não comunicação efetiva desta informação. O significado clínico desta omissão, no entanto, dependerá do medicamento 
omitido e do estado clínico do doente (ex. a omissão de antiepiléticos ou agonistas beta-adrenérgicos e o risco de síndromes de retirada com aparecimento de sintomas ou ainda síndromes de abstinência ou de efeito rebound). Estas omissões podem muitas vezes adiar ou mesmo impedir a identificação de diagnósticos provocados pelo uso de um determinado medicamento que pode ter causado direta ou indiretamente a admissão hospitalar. ${ }^{18}$

Os dados acerca do acesso, obtenção e registo da informação terapêutica e suas alterações demonstraram, portanto, a ocorrência de falhas no processo de documentação e salientam a necessidade de protocolos de registo da informação terapêutica, assim como o desenvolvimento e otimização de ferramentas que apoiem o processo de reconciliação, com melhor sistematização desta informação, tornando-a mais facilmente transferível e comunicável durante e após o internamento.

O processo de reconciliação terapêutica, no que concerne à obtenção e registo da informação terapêutica nas fontes de dados consultadas, levou em média 41,8 \pm 8,4 minutos por doente, o que é semelhante ao encontrado em estudos com as mesmas características como o de Giannini et al (2019), com tempo médio de 47,0 \pm 18,0 minutos num serviço de Medicina Interna na Suíça. ${ }^{19} \mathrm{O}$ tempo do processo de reconciliação terapêutica pode depender de diferentes fatores. A disponibilidade da informação fornecida pelo doente está ligada ao conhecimento deste acerca de seu regime terapêutico ou ainda ao seu estado clínico. Nestas situações, o familiar ou instituição que o acompanha são fontes de informação a serem consideradas. No entanto, muitas vezes a dificuldade de contacto com estes ou ainda o seu pouco conhecimento sobre a terapêutica do doente comprometem a disponibilização atempada ou completa das informações. ${ }^{19}$ Neste contexto, listas ou mesmo os medicamentos trazidos fisicamente ao hospital, assim como os registos eletrónicos dos cuidados primários, podem auxiliar a colheita de dados. Estes fatores dependem, porém, de disponibilidade de acesso e atualização, o que condiciona os recursos necessários e influenciam na realização ou não da reconciliação dentro das primeiras 24 horas de admissão.

Conforme observado nos resultados (Fig. 3), ao se tratar de um processo que precisa de ações coordenadas e integradas, vários recursos podem influenciar a reconciliação terapêutica, tais como a regulamentação vigente no país, o processo em si, as pessoas envolvidas (incluindo os profissionais, doentes e cuidadores), a gestão local e dos outros serviços, as ferramentas disponíveis e ainda a formação dos envolvidos. Toda esta junção de fatores exige um tempo que muitas vezes o profissional não tem, nomeadamente num contexto de admissão hospitalar, o que reforça a importância do mapeamento do processo, com identificação de pontos-chave e ações de intervenção baseadas na contribuição da tecnologia e das ações compartilhadas dentro da equipa de saúde.

A partir dos recursos prioritários identificados, agrupámos oportunidades de otimização em quatro tópicos cen- trais que poderiam tornar a reconciliação terapêutica mais aplicável no serviço, com suavização dos pontos frágeis identificados, como a falta de registos eletrónicos robustos (ex. PDS) ou mesmo a frequência da omissão como a categoria de discrepância mais prevalente. São estas as oportunidades: a otimização das fontes de dados disponíveis - melhoria na qualidade da BPMH e ampliação do acesso à informação clínica; normalização e informatização do processo - integração da informação terapêutica da admissão à alta hospitalar com diminuição de informações duplicadas, confusas e padronização dos registos; atuação multidisciplinar - definição de responsabilidades, ações integradas e maior envolvimento dos serviços farmacêuticos hospitalares; definição de grupos prioritários - baseados no mapeamento do perfil de doentes e discrepâncias e consequente estratificação de risco. As oportunidades identificadas, ou também relatadas na literatura como fatores facilitadores, corroboram os resultados de outras análises realizadas na transição de cuidados, que buscam identificar estratégias de estruturação de práticas seguras de medicação. ${ }^{20,21}$

Muitas instituições não conseguem dispor da equipa necessária para implementação da reconciliação de forma ampliada, o que pode comprometer que as intervenções sejam bem-sucedidas. ${ }^{22}$ Neste cenário, a gestão dos serviços acaba por recear a disponibilidade de recursos, uma vez que os benefícios não são suficientemente claros. Isto, por sua vez, acaba por impedir uma observação clara do impacto desta ferramenta na prática clínica, impedindo a visualização dos resultados esperados ao mesmo tempo que dificulta a sua viabilização. Assim sendo, estudos-piloto como este podem contribuir neste sentido por meio da identificação de prioridades e consequentemente melhor direcionamento dos recursos já disponíveis.

Uma vez que a implementação de um processo de reconciliação incompleto pode acabar por comprometer a segurança do doente ao invés de garanti-la, ${ }^{23}$ conhecer os fluxos e procedimentos existentes é essencial para promover ações consistentes e que permitam avanços. ${ }^{22}$ Por isso, identificar o perfil do serviço quanto às principais patologias, fármacos e discrepâncias permite ações mais focadas e resolutivas, baseadas em parâmetros reais.

\section{Contribuições e limitações}

Este estudo procurou identificar as práticas existentes, analisar pontos críticos e apontar estratégias que possam contribuir para este e outros serviços com caraterísticas semelhantes. Tem como limitações a ausência na equipa clínica de um farmacêutico atuante nos serviços clínicos, assim como a falta de um histórico ou grupo de comparação. A validação do método proposto noutros serviços, assim como a aplicação das otimizações discutidas, são pontos que a partir deste projeto podem ser explorados mais profundamente no futuro.

\section{Implicações clínicas}

Para implementação da reconciliação terapêutica na 
prática clínica, os principais recursos deverão estar voltados para o planeamento e estruturação do processo com estabelecimento de protocolos, responsabilidades e monitorização com indicadores de qualidade, assim como para a robustez das bases de dados e sistemas de informação clínica disponíveis, sua integração, atualização e acesso entre os serviços. Por fim, pode-se destacar ainda a necessidade da disponibilidade e atuação colaborativa da equipa multidisciplinar, assim como o envolvimento do doente ou do seu representante no cuidado transicional, visando um processo menos dispendioso e que, de facto, contribua para a segurança do doente.

Mais do que um critério de acreditação por si só, que por vezes pode trazer um peso burocrático e ser dispendioso, a reconciliação terapêutica deve ser vista e tratada com um objetivo global de segurança do doente não somente durante o internamento hospitalar, mas sim durante toda a continuidade do cuidado, o que inclui o próprio domicílio do doente, instituições sociais, centros de saúde, farmácias comunitárias e outros espaços de saúde.

\section{CONCLUSÃO}

Os resultados encontrados permitiram concluir a existência de fragilidades no que diz respeito à transmissão e registo da informação terapêutica na interface cuidados primários/hospitalares, assim como nos processos hospitalares internos. O melhor acesso às fontes de dados, o aperfeiçoamento dos métodos de documentação da informação terapêutica e suas alterações e a atuação interdisciplinar são pontos-chave a ser considerados na otimização e aplicabilidade da Reconciliação Terapêutica neste serviço.

\section{AGRADECIMENTOS}

Os autores agradecem ao diretor do Serviço de Medicina Interna, Armando de Carvalho, pelo apoio na realização deste estudo.

\section{REFERÊNCIAS}

1. Direção-Geral da Saúde. Norma 018/2016: Reconciliação da Medicação. 2016. [consultado 2021 nov 18]. Disponível em: https:// www.dgs.pt/directrizes-da-dgs/normas-e-circulares-normativas/norman-0182016-de-30122016.aspx.

2. World Health Organization. Medication Safety in Transitions of Care.; 2019. [consultado 2021 nov 18]. Disponível em: https://www.who.int/ publications/i/item/WHO-UHC-SDS-2019.9.

3. Institute for Healthcare Improvement. How-to guide: prevent adverse drug events by implementing medication reconciliation. 2011. [consultado 2021 nov 18]. Disponível em: http://www.ihi.org/resources/ Pages/Tools/HowtoGuidePreventAdverseDrugEvents.aspx.

4. World Health Organization. The High5s Project-Standard Operating Protocol - assuring medication accuracy at transitions in care. 2014. [consultado 2021 nov 18]. Disponível em: https://www.who.int/initiatives/ high-5s-standard-operating-procedures.

5. Michaelsen M, McCague P, Bradley C, Sahm L. Medication reconciliation at discharge from hospital: a systematic review of the quantitative literature. Pharmacy. 2015;3:53-71.

6. Mansukhani RP, Bridgeman MB, Candelario D, Eckert LJ. Exploring transitional care: evidence-based strategies for improving provider communication and reducing readmissions. P T. 2015;40:690-4.

7. Oliveira J, Cabral AC, Lavrador M, Costa FA, Almeida FF, Macedo A, et al. Contribution of different patient information sources to create the best possible medication history. Acta Med Port. 2020;33:384-9.

\section{CONTRIBUTO DOS AUTORES}

TCS: Desenho de estudo, colheita de dados, análise e interpretação de dados, redação e aprovação final do manuscrito.

PD: Desenho de estudo, colheita de dados e revisão crítica do manuscrito.

CAC: Colheita de dados, análise e interpretação de dados e revisão crítica do manuscrito.

JF: Desenho de estudo e revisão crítica do manuscrito.

ML: Análise e interpretação de dados e revisão crítica do manuscrito.

JO: Revisão crítica do manuscrito.

IVF: Desenho de estudo, revisão crítica e aprovação final do manuscrito.

MR: Desenho de estudo, análise e interpretação de dados, revisão crítica e aprovação final do manuscrito.

MC-B: Desenho de estudo, análise e interpretação de dados, revisão crítica e aprovação final do manuscrito.

\section{PROTEÇÃO DE PESSOAS E ANIMAIS}

Os autores declaram que os procedimentos seguidos estavam de acordo com os regulamentos estabelecidos pelos responsáveis da Comissão de Investigação Clínica e Ética e de acordo com a Declaração de Helsínquia da Associação Médica Mundial atualizada em 2013.

\section{CONFIDENCIALIDADE DOS DADOS}

Os autores declaram ter seguido os protocolos do seu centro de trabalho acerca da publicação de dados.

\section{CONFLITOS DE INTERESSE}

Os autores declaram não ter conflitos de interesses relacionados com o presente trabalho.

\section{FONTES DE FINANCIAMENTO}

Não houve qualquer fonte de financiamento.

8. Tam VC, Knowles SR, Cornish PL, Fine N, Marchesano R, Etchells EE Frequency, type and clinical importance of medication history errors at admission to hospital: a systematic review. CMAJ. 2005;173:510-5.

9. Buckley MS, Harinstein LM, Clark KB, Smithburger PL, Eckhardt DJ, Alexander $\mathrm{E}$, et al. Impact of a clinical pharmacy admission medication reconciliation program on medication errors in "high-risk" patients. Ann Pharmacother. 2013;47:1599-610.

10. Anderson LJ, Schnipper JL, Nuckols TK, Shane R, Le MM, Robbins K, et al. Effect of medication reconciliation interventions on outcomes: a systematic overview of systematic reviews. Am J Health Syst Pharm. 2019;76:2028-40.

11. Temido H. Internamento em Medicina Interna: evolução em 20 anos num hospital universitário. Med Interna. 2018;25:275-9.

12. Gleason KM, McDaniel MR, Feinglass J, Baker DW, Lindiquist L, Liss D, et al. Results of the Medications at Transitions and Clinical Handoffs (MATCH) Study: an analysis of medication reconciliation errors and risk factors at hospital admission. J Gen Intern Med. 2010;25:441-7.

13. Pippins JR, Gandhi TK, Hamann C, Ndumele CD, Labonville SA, Diedrichsen EK, et al. Classifying and predicting errors of inpatient medication reconciliation. J Gen Intern Med. 2008;23:1414-22.

14. Bell CM, Rahimi-Darabad P, Orner AI. Discontinuity of chronic medications in patients discharged from the intensive care unit. J Gen Intern Med. 2006;21:937-41.

15. Abu Farha R, Abu Hammour K, Al-Jamei S, AlQudah R, Zawiah M. 
The prevalence and clinical seriousness of medication discrepancies identified upon hospital admission of pediatric patients. BMC Health Serv Res. 2018;18:966.

16. Mongaret C, Quillet P, Vo TH, Aubert L, Fourgeaud M, Michelet-Huot E, et al. Predictive factors for clinically significant pharmacist interventions at hospital admission. Medicine. 2018;97:e9865.

17. Karaoui LR, Chamoun N, Fakhir J, Abi Ghanem W, Droubi S, Diab Marzouk AR, et al. Impact of pharmacy-led medication reconciliation on admission to internal medicine service: experience in two tertiary care teaching hospitals. BMC Health Serv Res. 2019;19:493.

18. Digiantonio N, Lund J, Bastow S. Impact of a pharmacyled medication reconciliation program. P T. 2018;43:105-10.

19. Giannini O, Rizza N, Pironi M, Parlato S, Waldispühl Suter B, Borella P, et al. Prevalence, clinical relevance and predictive factors of medication discrepancies revealed by medication reconciliation at hospital admission: prospective study in a Swiss internal medicine ward. BMJ Open. 2019;9:e026259.

20. Redmond P, Munir K, Alabi O, Grimes T, Clyne B, Hughes C, et al. Barriers and facilitators of medicines reconciliation at transitions of care in Ireland - a qualitative study. BMC Fam Pract. 2020;21:116.

21. Linden-Lahti C, Holmström AR, Pennanen P, Airaksinen M. Facilitators and barriers in implementing medication safety practices across hospitals within 11 European Union countries. Pharm Pract. 2019;17:1583.

22. Patel E, Pevnick JM, Kennelty KA. Pharmacists and medication reconciliation: a review of recent literature. Integr Pharm Res Pract. 2019;8:39-45.

23. Rungvivatjarus T, Kuelbs CL, Miller L, Perham J, Sanderson K, Billman $\mathrm{G}$, et al. Medication reconciliation improvement utilizing process redesign and clinical decision support. Jt Comm J Qual Patient Saf. 2020;46:27-36. 